\title{
Partial Duration Trend Analysis for Yearly Rainfall and its Sequential Distribution: a Conceptual Model
}

\author{
R.C. Yadav ${ }^{*}$ and S.C. Saxena
}

\author{
Central Soil and Water Conservation Research and Training Institute, Research Centre, Chhalesar, Agra-282006 Uttar \\ Pradesh, India
}

\begin{abstract}
In this study a new conceptual model for partial duration series analysis was applied to determine the likely rainfall of the monsoon season and the pattern of rainfall events distribution. Example case study was carried out with rainfall data of semiarid area, Agra, India, experiencing extremes of yearly rainfall; ranging between high (1200 mm) to low $(300 \mathrm{~mm})$. The coefficient values for the exponential decay function $(\mathrm{K})$ were computed for all decays of partial duration rainfall serises. Using the range of values of $\mathrm{K}$ and the yearly rainfall amounts further optimization produced the best results on the basis of the minimum Chi square value. The optimum $\mathrm{K}$ value was 0.92 . Using this $\mathrm{K}$, yearly rainfall values were predicted and compared with rainfall received for the validation period (1988-2002), not included in determination of optimum value of K. All the predicted yearly rainfall values were bit higher than observed ones. Further, for matching the predicted values with observed rain fall values, correction equations were also developed.

For distribution of rainfall events in the year, successive rainfall event that could have preceded at any time were contrasted with those of the past years with corresponding total rainfall values. As the rainy season progresses, the matching rainfall distribution of the corresponding rainfall year becomes the rainfall distribution pattern for year under the forecast; enabling selection of crops and cropping patterns before the onset of rainy season and carrying out strategic planning for successful agriculture under the changing scenario of rainfall pattern.
\end{abstract}

Keywords: Crop planning, drought and flood, forecasting, partial duration series, rainfall distribution, trend analysis, yearly rainfall.

\section{INTRODUCTION}

Forecasting of rainfall enables carrying out preplanning and preparations for agricultural activities. The ability to form good forecast is highly appreciated throughout the world. Statements are made about future events, patternizing the forecast closely on previous occurrences and under assumption that the future will be similar to the past. Rainfall is the most important parameter in agriculture in arid and semiarid areas, because it is the only source of water. Excess of rainfall may cause flood, while deficit or uneven distribution of rainfall lead to occurrence of agricultural droughts. Such trend analyses of short term cyclic pattern are utilized in Israel.

Agricultural practices particularly rainfed farming are based on rainfall and sequential distribution, where the partial duration trend analysis will play important decisive role in crop planning. Agricultural crop yields are dependent on the amount and distribution of rainfall events. This new conceptual model for partial duration trend analyses will enable to know future occurrence of rainfall that will make anyone get prepared and organize means to cope up with different

\footnotetext{
*Address correspondence to this author at the Central Soil and Water Conservation Research and Training Institute, Research Centre, Chhalesar, Agra-282006 Uttar Pradesh, India;

Tel: +91 562 6544599; Fax: +91 562 2883657;

E-mails: ramcyadav@ rediffmail.com,rcyadaveit@gmail.com
}

situations of rainfall aberrations. Accurate forecasting through such partial duration trend analyses will help carrying out planning for agriculture in any given area. The objective of this study was to carry out the partial duration trend analyses of yearly rainfall for generating knowledge before onset of the coming monsoon season with respect to likely yearly rainfall total and event distribution with a view to developing method of forecasting for strategic planning for successful agriculture. Data of research centre's observatory at Agra were used to exemplify the method and draw meaningful conclusions for the study region.

\section{EXISTING FORECASTING METHOD SCENARIO}

In the past attempts were made to devise simple methods and procedures by utilising statistical methods to forecast the future events pertaining to probability and amount of the rainfall etc. Generally, observed rainfall data of the event/season/year etc. are tabulated in ascending or descending serieses and return periods are analysed by Weibull's method $[1,2]$. Several statistical probability distributions namely normal distribution, $\log$ normal distribution, $\log$ Pearson III distribution, and Gumble distribution etc were studied by various researchers and predicted value were compared with the observed trend of probability distribution derived by Weibull's method [2-4]. Largely, average rainfall value matches with the values predicted by log normal distribution. The non comparative values occur at the extreme 
ends of lowest and highest rainfall values. On the other hand, the extreme values were invariably found fitting well with the predicted values by Gumble's method. In all the predictions, amount and probability distribution etc. were claimed to match with the observed value, but it does not give any clue for the sequence of the occurrences of the rainfall events. Thus, the utility of such predictions is limited in agro hydrology.

Marcov Chain is a known method to predict values of random variable based on the values of the earlier time period. The Marcov Chain model is extensively used to study spell distribution and other properties of rainfall occurrences [5]. Long term frequency behavior of wet and dry weather probability of occurrence of daily and weekly precipitations [5-7] were made with Marcov Chain model. However, for such predictions of yearly rainfall and subsequent sequence of rainfall events that actually will occur during the predicted year or weeks cannot be forecasted. The present study was aimed at fulfilling these research gaps for forecasting of yearly rainfall and distribution of occurrence of rainfall events by the new conceptual model of partial duration series trend analyses.

Studies [8] showed that climate variability (good or bad monsoon years) can influence the agricultural productivity in India by as much as $20 \%$. The forecasts are found with variable successes. Medium Range Weather Forecasting is carried out at National Centre for Developing Appropriate Operational Agro-meteorological models at Noida, national capital region New Delhi, India. Efforts are being made at the Centre to develop by any simple workable model the forecast for any given region. Thus, simple and accurate prediction measures are always desirable. This study aimed at enabling the farmers to take weather based decision in agriculture for efficient utilization of the positive influences of the weather resources, minimize the risk and adverse effects on crop productivity.

\section{ANALYTICAL CONSIDERATION}

\subsection{The Conceptual Model}

There can be several types of hydrologic years with rainfall comparable to the predicted rainfall. For the normal pattern of distribution of rainfall about 30 years rainfall data are utilized. Thus, the type of hydrologic year for any site or region can be determined based on variability in ranges of one standard deviation or two standard deviations on either side of the mean values. The internal distribution of the rainfall during the year can be decided by seeing the early, normal or delayed occurrence of rainfall. The year with comparable pattern of initial rainfall is taken to represent rainfall distribution for the likely successive part of the rainy season. In selection of annual rainfall, the position of the year in the cyclic pattern and consideration of initial rainfall events in the year lead to, respectively, determine annual rainfall and its event wise distribution.

\subsection{Partial Duration Series}

The cyclic patterns ranging from high to low in long term yearly rainfall distributions can be isolated. The cyclic peri- ods may be 3 to 5 or 3-8 or even 3-10 years. That means droughts occur at places once in three years to once in five years, once in eight years or are increasing due to global warming and climate change. There can be several series at any location depending on the geographical setting of weather of site under consideration. It is possible to derive the decay functions coefficient for individual cycles of any duration those will emerge in the long term data arrays. Since the cyclic pattern duration cannot largely be controlled, any attempt to standardize the duration of cycle appears to be not possible for much duration.

\subsection{The Decay Function}

The cyclic tendency of variation of rainfall is derived by a decay function [9] as

$\mathrm{I}_{\mathrm{t}}=\mathrm{I}_{0} \mathrm{~K}^{\mathrm{t}}$

Where $I_{0}$ is the initial value of the antecedent year precipitation, $I_{t}$ is the reduced value $t$ year later and $K$ is decay/recession factor ranging normally between 0.85 to 0.98 . Letting t equal to one gives $\mathrm{I}_{1}=\mathrm{KI}_{0}$.

The decay coefficients can be optimized for any region. For a given cycle, any year's rainfall can be determined by the decay function.

\section{MATERIALS AND METHOD}

\subsection{Delineation of Cyclic Patterns}

The long term rainfall data (1963-2002) from the meteorological observatory functioning since 1958 at Central Soil and Water Conservation Research and Training Institute, Research Centre's Research Farm, Chhalesar, Agra (27 $10^{\circ} \mathrm{N}$ and of $78^{\circ} 02^{\prime} \mathrm{E}$, msl $169 \mathrm{~m}$ ), India, shown in Fig. (1) were utilized for the study. For the rainfall variations the partial duration based cyclic patterns as occurred for Agra 3-5 years were isolated. The highest and lowest rainfall and intermediate values were patternized. The rainfall data for 26 years (from 1963 to 1987, both years inclusive) were utilized for the development of trend analysis.

\subsection{The Trend Analysis}

The rainfall for any year is equal to the previous year's rainfall multiplied by the index $\mathrm{K}$ eq 1 . By using this index it was possible to predict rainfall of the coming year based on that of the previous years. Taking full cognizance of cycle and position of the year in question, it was possible to predict the rainfall to a high degree of accuracy.

\subsection{Optimization of Index K}

Optimization was carried out by way of working out by the index of dispersion test criterion $\chi^{2}$ (Chi square test) by the eq.

$\chi^{2}=\sum(\text { observed }- \text { expected })^{2} /$ expected

By taking different successive values of $\mathrm{K}$ from 0.85 to 0.95 , the predicted rainfall was compared with the observed ones and $\chi^{2}$ values were computed. A graphical relationship 


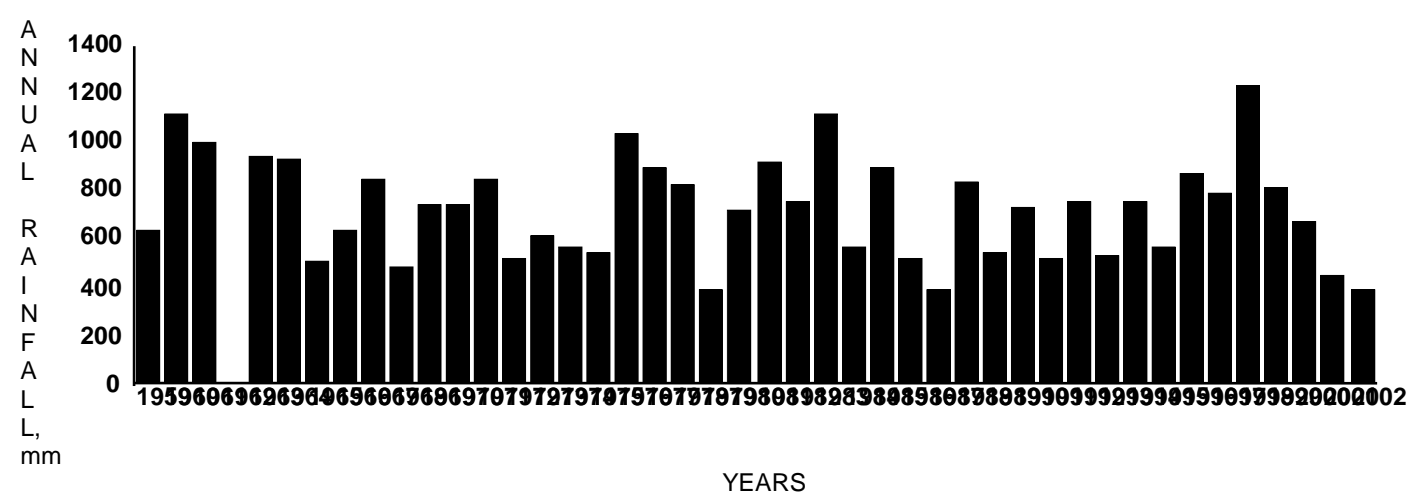

Fig. (1). Long term yearly rainfall of CSWCRTI Res. Centre Agra used for the study.

was developed (Fig. 2). The optimum value of $\mathrm{K}$ derived with the data for the years 1963-1987 was 0.92 .

\subsection{Validation for Rainfall Prediction}

The model was validated by utilizing annual rainfall data from (1988-2000) which were not included in the formulation of the model. By doing this, a forecast is always positive i.e. observed rainfall is more than the predicted value (Fig. 3). The predicted rainfalls of years needed further adjustment vide eqs. 3-5 to match with the observed values. The fact that observed rainfall is more than the predicted rainfall ensures a crop planning based successful crop production. The guidelines for different hydrologic years are as under:

$\mathrm{O}=\mathrm{P} \mathrm{mm}$ for normal year

$\mathrm{O}=\mathrm{P}-200 \mathrm{~mm}$ for extremely wet rainfall year

$\mathrm{O}=\mathrm{P}+100 \mathrm{~mm}$ for extremely drought year

In which $\mathrm{O}$ is observed rainfall of the year and $\mathrm{P}$ is the predicted rainfall of the forecast values for the same year. As indicated earlier, the position of year under forecast is known.

\subsection{Rainfall Pattern in the Identified Year}

Once rainfall of the year was ascertained, a corresponding rainfall matching year from the past record was selected in conformity with the hydrologic situation of the year. As season approached, a real pattern of the distribution of the rainfall whether early start or late start and early withdrawal or late withdrawal within the year was decided. This type of

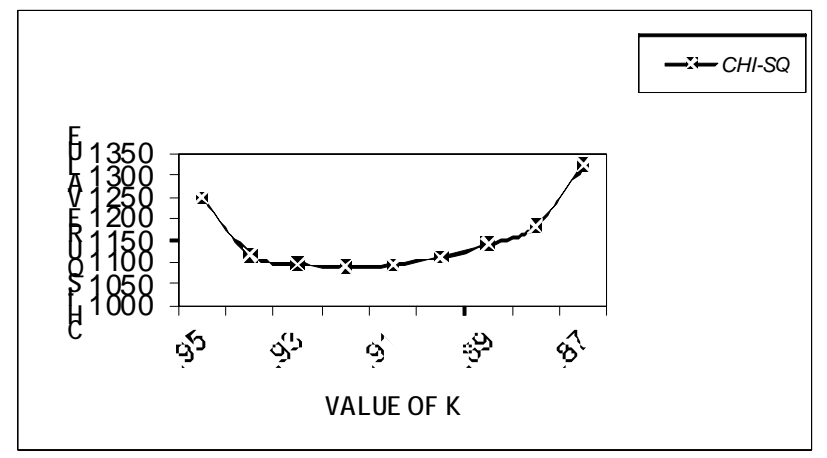

Fig. (2). Variation of values computed with different values of $K$ in eq. 1 for optimization. selection enabled forecasting of event distribution required for crop planning for the year.

\section{RESULTS}

\subsection{Annual Rainfall Forecast}

It is possible to predict annual rainfall by the model of trend analysis (Fig. 3). The prediction of yearly rainfall for normal and average years results are relatively comparable to observed rainfalls making the prediction very certain. The predicted annual rainfall values in the wet years (surplus and flood years) become more than observed. Hence the predicted rainfall values are reduced vide eq. 4 for matching the same with the observed rainfall. However, during the deficit extreme year, predicted rainfall is less than the observed one. In accordance with the extreme of variation of low rainfall appropriate value is derived by eq. 5 to arrive at the rainfall of the drought year. Thus, for prediction it requires to know position of hydrologic years that indicates the hydrologic characteristics and accordingly the fore mentioned adjustments vide eq. 4 and eq. 5 is carried out to arrive at final value of the corresponding year.

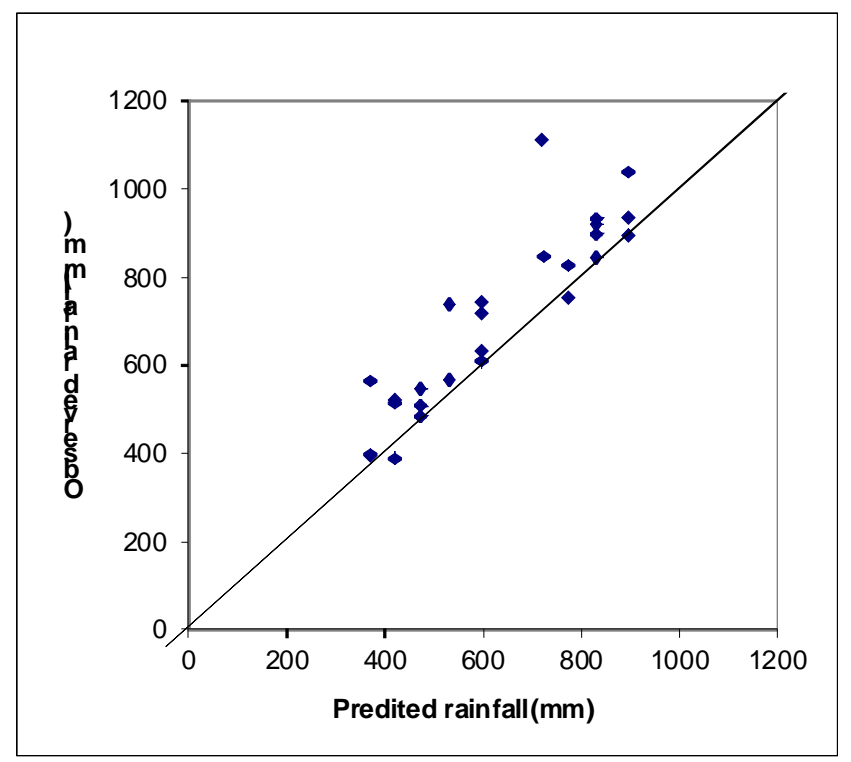

Fig. (3). Comparison between predicted and observed rainfall values. 

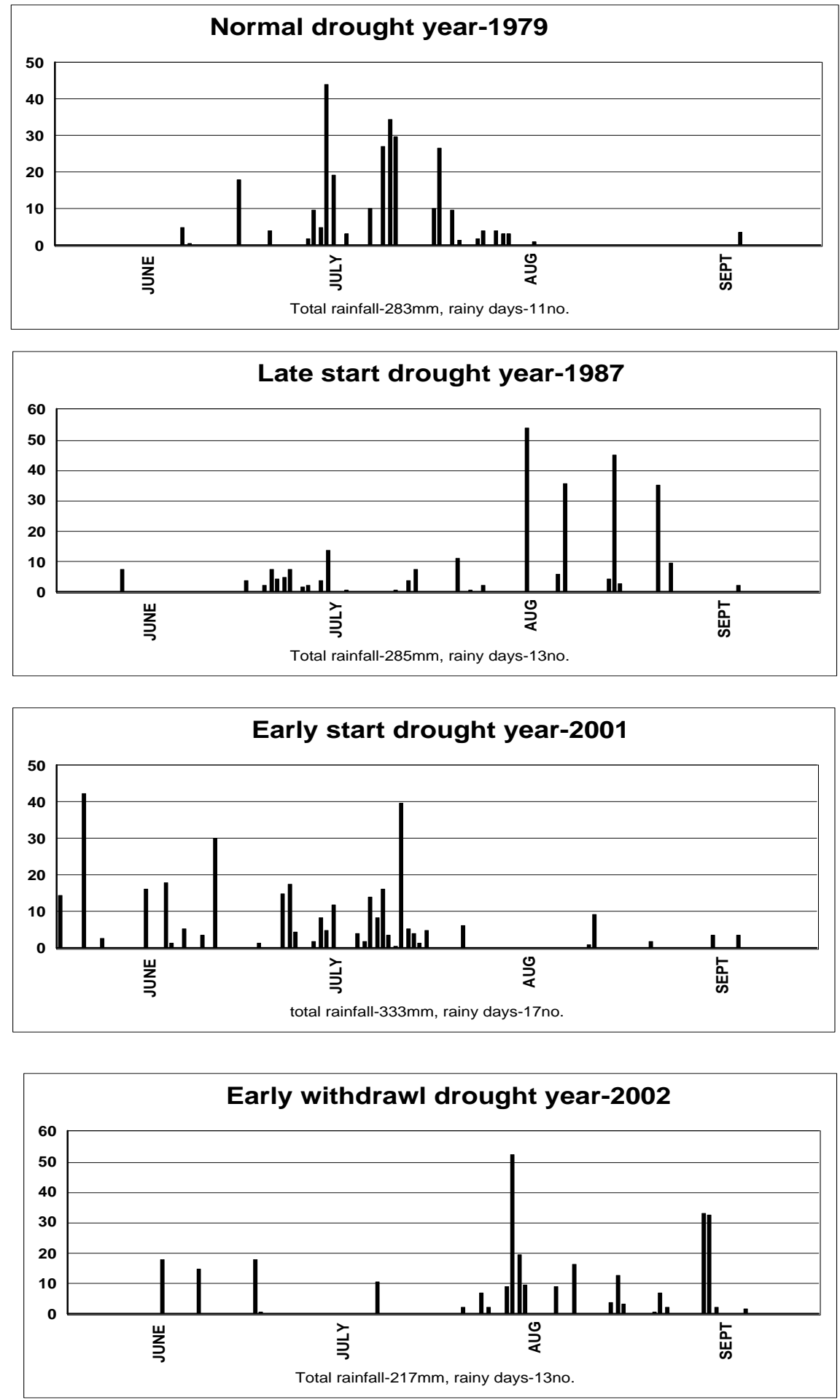

Fig. (4). Variable Rainfall-Pattern during drought year.

\subsection{Forecast of Rainfall Pattern}

Annual rain for the year 2002 was predicted to be a extreme drought year. Based on forecast of annual rainfall the pattern of rainfall distribution in the year 2002 was forecast to occur similar that of year, 1987.

The rainfall predicted for Agra during the year, 2002 was found matching very well with the observed rainfall in 1987.
It was predicted to be drought year with rainfall of $300 \mathrm{~mm}$. Till $16^{\text {th }}$ of July 2002 , there was no rainfall and that made almost certain that total rainfall during the rainy season i.e. July-September would not be more than $300 \mathrm{~mm}$. Year 2002 rainfall marched parallel to the rainfall that occurred in the year 1987, which was a very severe drought year (Fig. 4). After the severe drought year, the cycle reverted to high rainfall year. Accordingly, the prediction enabled carrying out 
forecast for the next year i.e. 2003 to be with rainfall more than normal i.e. resulting in situation of surplus or flood year. As predicted the year 2003 was a surplus rainfall year (Fig. 4). The rain fall distribution matched very well with the predicted rainfall for the year. Thus, it is possible to predict yearly rainfall and distribution of rainfall spells.

The higher values of predicted rainfall for the validation years establish that the forecasting is successful and it gives idea of rainfall that will occur for certain. Now for exact comparison of the predicted versus actually observed rainfall value's correction equations are to be used as the pattern of rainfall of the year is known to be in the range for the year viz surplus or flood year, normal year or the drought year. Thus, the study has produced the desirable quality and level of accuracy of prediction. Use of equations for comparing predicted versus the observed values can be further attempted by Lorenz curve analysis for measuring the inequality [10] in the income distribution in socio-political scenario in economic growth build up and income distribution to derive level of poverty.

\subsection{Crop Planning Based on Forecast}

The prediction of annual rainfall and its distribution enabled carrying out crop planning well in advance and making necessary preparations for inputs and agricultural operations. Since the predicted year 2002 was an extremely drought year, the further planning is described. Rainfall distribution study from the analysis of rain gauge charts of different typical drought years revealed the occurrence of three distinct types of situations viz. (i) drought year with early start and early withdrawal (ii) drought year with late start and late withdrawal of rains (iii) drought year with medium start and medium withdrawal. At Agra annual normal rainfall is 740 $\mathrm{mm}$ with extremes of minimum 300- maximum $1200 \mathrm{~mm}$ and the drought year occurs once in 3-5 years.

In the year 2002 up to the end of August total rainfall was about $250 \mathrm{~mm}$. In the rainy season from June 16 to August 29 the total rainfall was $210 \mathrm{~mm}$ while the rainfall that occurred in spells of $18 \mathrm{~mm}$ and $15 \mathrm{~mm}$ at week's interval in first and second week of June, 2002 helped carrying out summer ploughing. Rainfall that occurred in July (total $30 \mathrm{~mm}$ ) was insufficient to carryout sowing of rainy season (kharif crops). Further, in August light showers started from August 4 and till August 11, with total rainfall till then was only $20 \mathrm{~mm}$. On $12^{\text {th }}$ August $53 \mathrm{~mm}$ rainfall occurred that helped sowing of pearlmillet crop in this area. The normal sowing time for pearlmillet in the study region is first week of July. Thus, there was a delay of sowing of pearlmillet by $1-1 / 2$ months. Pearlmillet sown around $15^{\text {th }}$ of August matured by October provided there was supplementary irrigation to carry over the crop. The pearl millet crop without supplemental irrigation produced about $20-30 \%$ of normal yield. When one supplementary irrigation was given to the crop, the yields of pearl millet crops in the area were about $2.5 \mathrm{t} / \mathrm{ha}$, which was very high yield. Under such delayed onset of rainfall it is advisable to the farmers to raise nursery of bajra (pearlmillet) with the use of pumping sets and transplant the seedlings at the establishment of rains. This practice will enable farmers to produce pearlmillet for their domestic consumptions. Otherwise, such delayed rainfall years may be a total failure year for production of pearlmillet in particular and pulses, in general. The pearl millet is main cereals for food in arid and semi arid countries of world in the southern hemisphere as well as countries in the tropics and semi arid tropics.

\section{DISCUSSION}

The trend analysis based model is found to enable precisely predict annual rainfall well before the onset of rainy season. Accordingly, based on the beginning of the season likely rainfall pattern is also found matching with observed rainfall. The trend analysis proved superior to other methods of probability distribution and other statistical analysis with regard to forecasting of occurrence of rainfall and its distribution. This forecasting would help planning of cropping pattern for the year and arranging necessary inputs well before time of sowing. By adjusting the cropping pattern adverse impact of droughts and floods can be mitigated to a larger extent.

The trend analysis can be considered as a rational approach as many processes follow a decay function [9]. It requires the establishment of series from highest to lowest. Thus, after establishment of series and the decay function has been patternised in this model preparation for forecasting, the rainfall fore casting becomes easy. It may be mentioned that the position of the year under the forecast is already known, which will be within the cycle of 3-5, 3-8 or 3-10 years. Nevertheless, the rain making processes is governed by many factors such as solar heat, presence of humidity, process of cooling by condensation and sublimation, and air that sweeps away entire process. Hence it is highly cumbersome to predict occurrence of such a complex process. In this situation it can be argued that even medium range forecasting fails so the trend analysis established in the present study can not be successful. Inspite of the facts of complex process, it was found to working well for prediction of rainfall during drought year (2002) in the study and for year with rainfall more than normal during the subsequent years. Such prediction and forecasts were made in the subsequent years and famers were advised for their cropping patterns in the region. Thus, it can be a dependable model for forecasting of rainfall and its distribution, as good as many complex analysis based forecastings are successful. The Lorenz curve analysis is known method to measure any inequality [10]. Likewise the success of this in forecasting of annual rainfall and the event distributions, such analyses can be conducted for many areas and hydrologic situations can be decided and graded on the basis of inequality of forecasted values in contrast with actually that will occur. However, this requires exhaustive and coordinated research investigations. This study has devised new method and demonstrated utility of forecasting of yearly rainfall and distribution of events in the entire season.

The statistical models for analyses of weather data have been stake and used worldwide. Skeptical attitude develop in the scientific community about failures of such predictions due to global warming and climate change. This is true for 
any sophistication based prediction as well. Once this partial duration analysis is utilized for capitalizing agro-hydrology at any site, further necessary improvements can be thought over depending on the situations. This fact of utilizing the present time in the best possible way is true for all time and for any advanced or simple analysis.

Even if the process of forecasting by the present model fails, there can be many midway corrections. Because agriculture is time sensitive, particularly, sowing time, ways and means can be adopted to complete sowing within the optimum sowing periods of crops. For example in the drought year with delayed onset of monsoon nursery raising of pearlmillet and transferring after one and half month could produce yield at par achievable during normal rainfall year. Conversely, during high rainfall year, the sowing of green gram needed deferment for about a month to avoid excess rainfall.

Recently new cultivation technology has been developed where this research will find greater application in devising cropping sequence for successful harvest [11]. Further, once crop sowing is carried out, mid season corrections will enable crop physiological and nutrient management for successful harvest, which otherwise remain uncertain. Thus, it will be possible to decide the strategy of crop and water management so that successful harvest can be acquired in any adverse climatic situation. Thus, an element of certainty is brought in agriculture in the present days of changing climate, which otherwise was regarded as mere helplessness. The situation of certainty will usher boost in productivity of rainfed agriculture in semi arid and arid areas. The successful harvest will enable reduction in dependency on the market and escape price rise. Food insufficiency triggers socio political instability and change of governance [10].

\section{SWOT ANALYSIS}

Strength, weakness, opportunity and threat analysis of the present study is carried out in the following.

\subsection{Strength}

The study has developed simple, organisable, feasible and scientifically correct approach for prediction of rainfall year, total rainfall and distribution of event before onset of monsoon season for rainy season crop planning and preparation of the facilities. The method developed in the study adds strength of knowledge about upcoming future and enables make necessary preparations for crop cultivation. The study also suggests the mid season correction even if the prediction fails for carrying out successful crop in the rainy season. This situation of knowledge enables rainfed farming get free from high degree of risks of crop failure. The severe most drought years can be managed by application of science and technology. This fore casting and pre planning will enable small and medium land holding families to produce food and get free from the stress of rise in the market price. Since food is the first priority, this type of agrarian development would help take out of food shortages and hunger. The changing climate bring food shortages situation in the arid and semiarid countries of world. Under this condition the fore cast and agro-technology [11] would sail the poor families get out of food shortage.

\subsection{Weakness}

The study has no technical weakness. Nevertheless, the climate is suffering from the adverse effect of global warming. The results presented in the study overcome the undue scientific criticism that many sophisticated predictions fail so what to talk of this conceptual prediction. Since data used was for the long term, comparison with observed values with predicted add full confidence on its accuracy and utility. It needs coordinated study and establishment as an alternative method for predictions in regions having features similar to that of Agra in the typical semi arid region.

\subsection{Opportunity}

The study provides a sound scientific basis for analyzing climatic data to assess the effect of climate change. The analysis will enable determining the region where climate change will be severe and what effect it will bring on hydrologic variables and its effect on the climate and period since when global warming became serious. The forecasting will enable people to store food for their family needs till the next harvest of crops. This will help people to escape from hunger and mal nutrition. The individual storage of adequate food will eliminate loss of food grain in the storage in the government, especially when the storage structures are not sufficient for safe keeping of food commodities from the previous harvest. In another study [12] the first researcher has established that such storage losses of food distort nitrogen cycle, which bring many adverse effects on peoples' health, water quality, water body and eutrophication etc.

\subsection{Threat}

There is no threat from applying the results of the partial duration series based fore casting study. However, some speculative hording of commodity by the traders may bring rise in the cost of food commodity till development of monsoon for short period. Strategic control measures can overcome such disadvantageous use of the forecasting in speculative hording of food commodities. Nevertheless, the gains from application of results overtake and overshadow the threat what so ever it may be.

\section{CONCLUSION}

Yearly total rainfall and pattern of rainfall events' occurrence forecasting model based on partial duration trend analysis is found to predict rainfall for two successive drought years as well as for more than normal year presented in this study and for beyond this period for Agra. This partial duration forecast enables carrying out advance crop planning and arranging inputs etc. for achieving sustainable agriculture in the region. Any failure in the prediction can be easily improved upon by mid season corrections. Thus, this study adds new dimension for management of agriculture, water resources, food and governance etc.

\section{CONFLICT OF INTEREST}

The authors confirm that this article content has no conflict of interest. 


\section{ACKNOWLEDGEMENTS}

The research was carried out at the centre. However, there was no specific allocation of research fund.

\section{REFERENCES}

[1] Linsley RK, Kohler MA, Paulhus JLH. Hydrology for engineers. McGraw Hill International Book Comp. 1982; 242-3,

[2] Chow VT. Hand book of applied hydrology. McGraw Hill Book Co. Inc.1964. Chapter 8, Section 1

[3] Hann CT. Statistical method in hydrology. East-West Press Pvt. Ltd., Delhi. 1994; 128-158.

[4] Kottegoda NT. Stadiastic water resources technology. Mac Milliam Press Ltd. London. 1980.

[5] Gabrial KR, Newongnn J. A Marcov chain model for daily rainfall occurrences at Tel Aviv, J.R. Met. Soc.; 1962: 88.

[6] Pandrinath N. Marcov Chain model probability of dry and wet weeks during Monsoon period over Andhra Pradesh, Mausam 1991; 42(4): 393-400.
[7] Victor US and Sastry PSN. Dry spell probability by Marcov Chain model and its application to crop development stage. Mausam, 1979; 30: 479-89.

[8] Sulochana G. Climates changes in agriculture - An Indian perspective. InAbrol YP., Gadgil S., Pant GB. [Ed] Climates variability and Agriculture, Narosa Publishing House, New Delhi, India, 1996; $1-18$.

[9] Rodriguez Iturbe I, Mejia JM. The design of rainfall networks. In time and space. Water Reso Res 1974; 10(4).

[10] Felix R, Sampat M, Amitava G, Rajendra NN. Contemporary Development Economics from Adam Smith to Amartya Sen, New Central Book Agency, Pvt Ltd, London, New Delhi.

[11] Yadav RC. The racy nature agriculture and other alike technologies: A technology contrast. Middle East Journal of Scientific Research, Dubai, 3013 published on line December.

[12] Yadav RC. Innovative application of scientific facts for arresting GHG- $\mathrm{N}_{2} \mathrm{O}$ and improvising lucrative ventures with enhanced land, water and nutrient use efficiency J Energy Environ Sci Photon 2014; 128: 486-520.

Received: May 26, 2014

Revised: July 27, 2014

Accepted: August 01, 2014

(C) Yadav and Saxena; Licensee Bentham Open.

This is an open access article licensed under the terms of the Creative Commons Attribution Non-Commercial License (http://creativecommons.org/licenses/by-nc/3.0/) which permits unrestricted, non-commercial use, distribution and reproduction in any medium, provided the work is properly cited. 